\title{
Incidence and distribution of injury among schoolchildren aged 11-15
}

\author{
CE Currie, JM Williams, P Wright, T Beattie, Y Harel
}

\begin{abstract}
Objectives-To measure the incidence and age and sex distribution of self reported experience of injuries in the preceding 12 month period among a representative national sample of Scottish schoolchildren and to validate the findings against other data sources.
\end{abstract}

Design-Self completed questionnaire administered in schools, April-June 1994.

Subjects-4710 pupils aged 11, 13, and 15 years drawn from a representative sample of 270 classes with returns from 224 classes ( $83 \cdot 2 \%$ completion rate).

Outcome measures-Number, type, site, and severity of injuries reported.

Results-41.9\% of pupils reported a medically attended injury, with injury incidence significantly higher in boys than in girls. Using the abbreviated injury scale (maximum abbreviated injury score) one third of injuries were either moderate or severe.

Conclusion-The incidence and distribution of self reported injury is consistent with estimates based on other data sources thus confirming the utility of this method of injury surveillance in this age group.

(Injury Prevention 1996; 2: 21-25)

Keywords: self completed questionnaires, incidence, self reporting, severity.

Childhood injuries have been highlighted as a cause for concern in recent government documents. ${ }^{1-3}$ In England and Wales half of all deaths of children aged 10-14 years are attributable to injuries. Injuries also cause one child in six to attend an accident and emergency department every year and account for a fifth of all paediatric admissions. ${ }^{4}$ Scottish mortality figures are even higher, and through the 1980 s this trend remained more or less static. ${ }^{5}$ National baseline information on the incidence of non-fatal injury in the population of school aged children is vital to the planning of preventative policies and programmes.

A current review of child injury prevention in Scotland concludes that there are 'serious limitations of existing epidemiological data and the associated difficulty of developing understanding of the aetiology of child accidents' ${ }^{6}$ Much injury data is specific to particular kinds of injury events - road traffic accidents, poisonings, and sports injuries, among others, and of necessity, the data are collected in different ways. Even within any one injury category, data from different sources, for example, hospital admissions, police and school records, may still be incompatible. ${ }^{7}$ It is not possible to combine this existing data to obtain an estimate of all injuries. One way of conceptualising the distribution of the full range of childhood non-fatal injury in the population is by means of a pyramid representing the severity of injury and care provided (figure). What is needed to achieve a population estimate of the full range of injuries is a comprehensive survey.

One such survey conducted recently in the USA produced national estimates of non-fatal injury rates among children and adolescents, and their relationship to sociodemographic, health, and behavioural factors. ${ }^{8}$ Injury data were derived from interviews with mothers who were asked about injuries sustained by their children in the past 12 months. Maternal reports must be employed when dealing with injuries during infancy. However, there is evidence that children of elementary school age often report injuries of which parents are not aware, thus for these older children, parents may not be able to report the full extent of injuries. ${ }^{9}$

Many childhood injuries do not result in hospital attendance and, in any case, admissions to hospital do not accurately reflect the community distribution of injury. ${ }^{10}$ In the above study, injuries were classified as severe if they resulted in hospitalisation, loss of activity, or absence from school for at least half a day. ${ }^{8}$ So even if we accept the adequacy of these definitions, severity of injury is not necessarily the criterion that determines hospitalisation. For example, availability of beds and social class both influence admission for all but the most serious injuries. ${ }^{11}$ Here we report measures of injury severity that have previously only been employed using clinical notes, namely the abbreviated injury scale

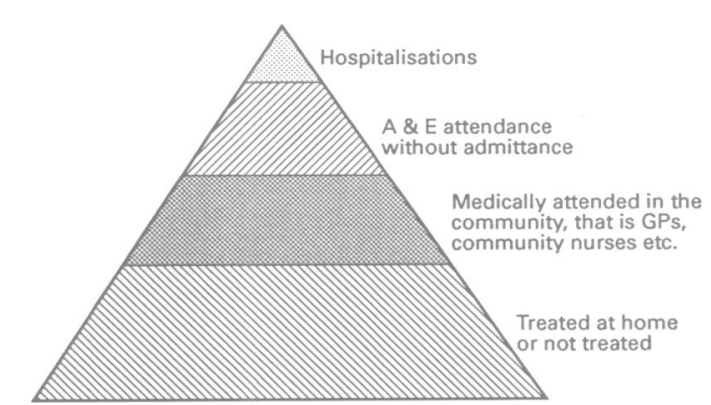

Schematic representation of childhood injury severity and resulting treatment ( $A \mathcal{E} E=$ accident and emergency department, $G P=$ general practitioner). 
(AIS). ${ }^{12}$ This allows for comparison with the proxy measures of severity commonly used in questionnaire based surveys.

For adolescents, asking them directly would seem to be a particularly appropriate means of data collection. This paper, therefore, provides self reported data on the incidence in the past 12 months of all non-fatal injuries in Scottish schoolchildren within an age range particularly at risk from injury, and compares these with estimates based on other data sources. Injury prevention strategies must be targeted at high risk subpopulations, such as adolescents, and also evaluated to ensure their effectiveness. Only with appropriate data can this be achieved.

\section{Subjects and methods}

The survey sample was stratified by region and based on the Scottish population of schoolchildren at the ages of 11,13 and 15 years (that is, primary year 7 , secondary year 2 , and secondary year 4). The study employed a clustered sample design where the primary sampling unit was the school class. This procedure is consistent with that used successfully in the Scottish World Health Organisation cross-national health behaviour in schoolchildren survey. ${ }^{13}$ The survey was conducted between April and June 1994. Children completed the questionnaires independently during a single school period (approximately 40 minutes) with the supervising teacher instructed to respond only to children's queries about procedure.

\section{SURVEY QUESTIONNAIRE}

The survey questionnaire was developed from a standardised instrument ${ }^{13}$ and comprised sections relating to injuries sustained over the past 12 months, demographic factors, social and psychological variables, and risk behaviours.

In the injuries section, children were initially presented with a list of injury types and asked whether they had experienced any of these in the past 12 months. These injury types characterise the full range of injuries experienced by children at these ages and were written in age appropriate terms. ${ }^{14}$ Children were next required to estimate the number of all injuries they had sustained over this time period. A filter question then asked 'Have you had to go to a doctor, a nurse or hospital because of any of these injuries?' The remaining questions, including a request for a description of the most severe injury sustained in the past 12 months (written in the child's own words), the timing and circumstances of the injury, were only

Table 1 Sample characteristics by age and sex

\begin{tabular}{llllll}
\hline & Total No & No of classes & Mean $(S D)$ age & No of boys & No of girls \\
\hline Age (years) & & & & & \\
11 & 1666 & 81 & $11 \cdot 72(0.31)$ & 836 & 830 \\
13 & 1504 & 71 & $13.67(0.32)$ & 740 & 764 \\
15 & 1540 & 72 & $15.63(0.33)$ & 754 & 786 \\
Total & 4710 & 224 & $13.63(1.64)$ & 2330 & 2380 \\
\hline
\end{tabular}

completed if the child reported a medically attended injury.

Descriptions of medically attended injuries were coded using the AIS. ${ }^{12}$ Where a child had written up to three injuries resulting from a single injury event each was coded independently and the maximum abbreviated injury score (MAIS) calculated. This is the highest single abbreviated injury score for an individual with one or more injuries and is used to described the total severity of injuries sustained from a single event. Coding was carried out by one medically experienced person trained in the use of the scale. All data was analysed by SPSS, using $\chi^{2}$ to assess the distribution of injury experience by age and sex. Relative risks were also calculated for sex differences in reported injuries. Regional weightings were applied to calculate the population estimates presented in the tables, but statistical tests were performed on unweighted data.

\section{Results}

Of the 270 selected classes, completed questionnaires were returned by 224 classes $(83.2 \%$ completion), a total of 4710 children (table 1 ).

\section{ALL INJURIES}

Of those children surveyed, 94.5\% (4465) reported having sustained an injury in the past 12 months, and more boys reported injuries than girls (boys $v$ girls, relative risk $(\mathrm{RR})=1.02,95 \%$ confidence interval $(\mathrm{CI})$ 1.01 to 1.04$)$. Of these, $8.8 \%$ (418) of children reported only bruises, $4 \cdot 2 \%$ (199) reported only cuts, and a further $17 \cdot 7 \%$ (837) reported both bruises and cuts. Overall, $41.9 \%$ (1911) of children reported having required formal medical attention from a nurse, a doctor, or at hospital for an injury.

The most commonly reported injuries were bruises and cuts followed by sprains; least common were poisonings and near drownings. The incidence of sprains and burns increased with age; head injuries resulting in loss of consciousness were most frequent among the 13 year olds; near drownings and unclassifiable other injuries were less frequent among older children (table 2).

Across all ages boys were more likely than girls to report a break or dislocation ( $R R=1.59,95 \%$ CI 1.38 to 1.83$)$; a sprain or strain $(1.22,1.14$ to 1.30$)$; cuts or bites $(1.06$, 1.02 to 1.09 ); that they had been knocked out $(2.58,2.00$ to 3.33$)$; near drownings $(1.96,1.37$ to $2 \cdot 80)$; and unclassifiable other injuries $(1.68$, 1.37 to 2.05 ). The exception to this global pattern was bruises, where girls showed a higher incidence $(\mathrm{RR}$ for boys $=0.96,95 \% \mathrm{CI}$ 0.94 to 0.99 ). No sex differences were found for burns or poisonings.

Across all ages $53.3 \%$ (1651) of children were injured three times or fewer, $36.2 \%$ (1121) were injured four to 10 times, and $10.5 \%$ (326) were injured 11 times or more in the past 12 months. More boys $(51 \cdot 1 \%)$ than 


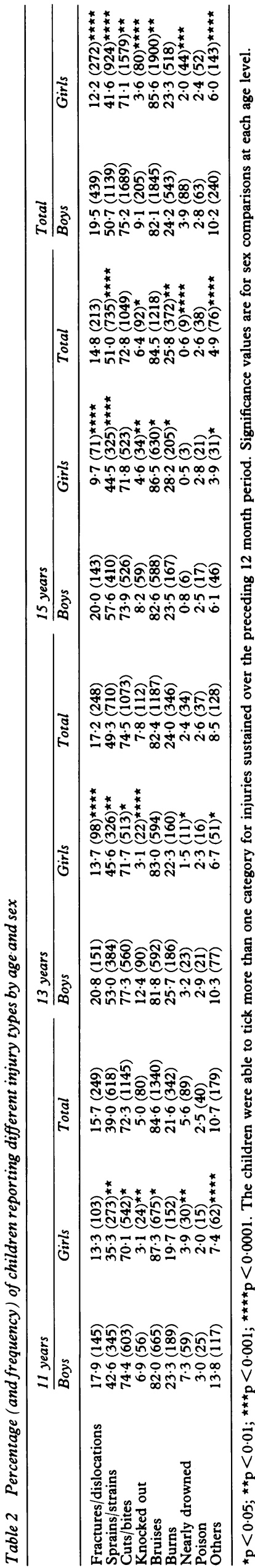

girls $(41 \cdot 7 \%)$ reported four or more injuries $\left(\chi^{2}=23.56, \mathrm{df}=2 ; \mathrm{p}<0.0001\right)$.

\section{MEDICALLY ATTENDED INJURIES}

The first injury reported by the child was analysed in detail, considering the site and type of injury independently because most children only identified one medically attended injury. Injuries to the upper and lower extremities occurred most often; least common were injuries to the neck/spine or the thorax/ abdomen. Head/face and external injuries significantly decreased with age, whereas neck/ spine and lower extremity injuries increased in frequency with age (table 3).

There were very few associations between sex and injury site, and the only significant sex difference in $R R$ was for head injuries, where boys presented more often than girls $(\mathbf{R R}=1.36,95 \%$ CI 1.06 to $1 \cdot 74)$.

The AIS scores were also grouped into: fractures and dislocations, sprains and strains, wounds, burns, and others. Fractures/ dislocations increased and wounds decreased with age. There was no association of the frequency of burns, sprains/strains, or other injuries with age (table 4).

Boys were more likely to have reported a medically attended injury than girls $(R R=1.3$, $95 \%$ CI 1.21 to 1.39). Analysis of sex differences for these types of injury showed that boys had a higher risk of sustaining wounds $(1.32,1.14$ to 1.54$)$. They had a lower risk of sprains or strains, however $(0.82,0.67$ to $0.99)$, and a lower risk of 'other' injuries ( 0.8 , 0.68 to 0.93 ). There was no sex difference in relative risk of fractures/dislocations or burns.

\section{SEVERITY SCORES}

MAIS was associated with age, signifying higher frequencies of more serious injuries in older children $\left(\chi^{2}=10.11, \mathrm{df}=4 ; \mathrm{p}<0.05\right)$. Analysis of MAIS showed no systematic associations with sex. Most boys and girls $(70 \%)$ reported minor injuries $($ MAIS $=1)$.

Other indices of injury severity were overnight stays in hospital and missed days of school. Boys were more likely to have spent a night in hospital (boys $=18.3 \%$; girls $\left.=12.4 \% ; \chi^{2}=11.71, \mathrm{df}=1 ; \mathrm{p}<0.001\right)$. Although there were no sex differences in missed days of school, a higher percentage of 13 year olds and 15 year olds than 11 year olds missed school due to injury $\left(\chi^{2}=10 \cdot 18, \mathrm{df}=2\right.$; $\mathrm{p}<0.01)$. The percentage of injured children staying overnight in hospital or missing days of school increased as a function of MAIS. This pattern was consistent for both girls and boys (table 5), and also held for each age group.

\section{Discussion}

This is the first survey to provide national baseline data on the incidence of non-fatal injuries over a 12 month period in schoolchildren. To date there has been little consistency in the literature concerning the appropriate cut off point for 'injury' (that is medical attention, 
hospital attendance, hospital admissions, etc). Consequently varying population injury rates and risk factors have been reported. ${ }^{15}$ Thus external validation of the population figures reported here requires careful examination of existing data from a range of sources.

Children's self reports confirm the pyramid model for injuries - $42 \%$ sought medical attention in some form for an injury, but $95 \%$ reported having sustained an injury of some kind in the preceding 12 months. These figures are high compared with hospital based injury statistics. For example, in 1991 Scottish children aged 5-14 years had a hospital admission rate of $1465 / 100000$ for non-intentional injuries. ${ }^{16}$ Hospital admissions correspond to the tip of the pyramid described in the introduction whereas our national figures are based on a broader definition of medical treatment by including attendance at an accident and emergency department and community medical treatment.

In England and Wales approximately $18 \%$ of the child population attend an accident and emergency department because of an injury. ${ }^{4}$ In Scotland the total figure for accident and emergency attendance of 5-14 year olds is difficult to obtain. However, the road injuries casualty rate, based on STATS 19 police motor vehicle accident records for 1991, was 483.6 / $100000 .{ }^{16}$ The home injuries rate, derived from the Home Accident Surveillance System in 1991 , was $5841 \cdot 1 / 100000,{ }^{17}$ and the leisure injuries rate, based on the Leisure Accident Surveillance System in 1991, was 153.5/1000. ${ }^{18}$ Of the South Glamorgan child population, an estimated $19.8 \%$ attended an accident and emergency department due to injuries annually (23.4\% of $10-14$ year olds). ${ }^{19}$ These attendance figures amount to approximately half of those who sought medical attention in our survey. Hospital attendance and admission surveillance system estimates of injury incidence are approximately doubled, however, when treatment by community based practitioners are also included. ${ }^{20}$

Table 3 Site of medically attended injury as a percentage (and frequency) of children by age

\begin{tabular}{|c|c|c|c|c|}
\hline Injury site & 11 years & 13 years & 15 years & Total \\
\hline $\begin{array}{l}\text { Head/face }{ }^{\star \star} \\
\text { Neck/spine } \\
\text { Thorax/abdomen } \\
\text { Upper extremity } \\
\text { Lower extremity }{ }^{\star \star \star \star} \\
\text { External }{ }^{\star}\end{array}$ & $\begin{array}{l}15 \cdot 8(106) \\
1.8(12) \\
0 \cdot 8(5) \\
25 \cdot 0(168) \\
28 \cdot 0(188) \\
13 \cdot 1(88)\end{array}$ & $\begin{array}{r}13 \cdot 5(86) \\
3.0(19) \\
1.0(6) \\
23 \cdot 4(149) \\
36.1(230) \\
11.0(70)\end{array}$ & $\begin{array}{c}9 \cdot 7(58) \\
4.3(25) \\
1.9(11) \\
25.9(155) \\
39.9(239) \\
7.8(47)\end{array}$ & $\begin{array}{c}13 \cdot 1(250) \\
3 \cdot 0(57) \\
1 \cdot 2(22) \\
24 \cdot 8(472) \\
34 \cdot 4(657) \\
10 \cdot 8(205)\end{array}$ \\
\hline
\end{tabular}

${ }^{\star} \mathrm{p}<0.05 ;{ }^{\star \star} \mathrm{p}<0.01 ;{ }^{\star \star \star \star} \mathrm{p}<0.0001$. Significance values are for comparisons between age groups.
The extent of community based practitioners' treatment of injuries is even more difficult to assess on a national level. The only UK based general practitioner study reported in the last five years estimated that $238 / 1000$ children in Oxford aged 10-15 years visited their general practioner because of an injury. ${ }^{21}$ Taken together, national and regional figures on hospital admission, attendances, and general practitioner based treatments match well with the $41.9 \%$ figure we have identified using the child self reports. Moreover there is also good agreement between our data and that of the American study that used maternal rather than child self report of medically attended injury. ${ }^{8}$ The authors reported an adjusted injury rate of $38 \%$ in an adolescent population, and in view of the likelihood of under reporting by mothers, ${ }^{9}$ this is a close match.

A second means of validating our data is to compare findings regarding injury severity and type. One problem with using the AIS to classify injury severity, in the context of a self report study, is the absence of clinical notes. In our case, this results in a likely underestimation of severity due to the fact that children usually provide insufficient detail and coding was conservative towards the least severe outcome.

Of the children who received medical attention, analysis of the MAIS showed that for approximately one third, their injuries were either moderate or serious. Good agreement is found with another UK study, where, of child attendances in an accident and emergency department, $71 \%$ require simple investigation and treatment, $23 \%$ require outpatient surgical treatment, $4.5 \%$ are admitted for observation, and $1.5 \%$ are admitted for treatment. ${ }^{19}$ Comparable figures have been reported from Dunedin in New Zealand. ${ }^{22-24}$ The most common types of medically attended injuries reported by Scottish schoolchildren (that is fractures/dislocations, wounds, and sprains/ strains) are the same as those found in New Zealand, as is their relative incidence at each age (with the exception of sprains/strains). ${ }^{22-24}$

Our sex differences are also consistent with the robust research finding that boys are more likely to report and present with an injury than girls. ${ }^{25}$ In general, boys had a higher rate of all injuries and medically attended injuries, but for individual injury types there was variation in numbers of boys or girls presenting for medical attention. This calls for further research into the factors determining which injuries are treated at home and which present for medical attention. In particular, the responses of parents and other responsible adults

Table 4 Type of medically attended injuries as a percentage (and frequency) of girls and boys

\begin{tabular}{|c|c|c|c|c|c|c|c|c|}
\hline & \multicolumn{2}{|l|}{11 years } & \multicolumn{2}{|l|}{13 years } & \multicolumn{2}{|l|}{15 years * } & \multicolumn{2}{|l|}{ Total } \\
\hline & Boys & Girls & Boys & Girls & Boys & Girls & Boys & Girls \\
\hline $\begin{array}{l}\text { Fractures/dislocations } \\
\text { Sprains/strains } \\
\text { Wounds } \\
\text { Burns } \\
\text { Others }\end{array}$ & $\begin{array}{l}20 \cdot 0(77) \\
13 \cdot 9(53) \\
38 \cdot 1(147) \\
5 \cdot 1(20) \\
22 \cdot 8(88)\end{array}$ & $\begin{array}{l}27 \cdot 0(77)^{\star} \\
16 \cdot 2(46) \\
28 \cdot 4(81)^{\star} \\
3 \cdot 6(10) \\
24 \cdot 9(71)\end{array}$ & $\begin{array}{l}28 \cdot 8(103) \\
16 \cdot 4(59) \\
30 \cdot 2(108) \\
4 \cdot 3(16) \\
20 \cdot 3(73)\end{array}$ & $\begin{array}{r}28.0(78) \\
21.9(61) \\
21.6(61) \\
4.7(13) \\
23.8(67)\end{array}$ & $\begin{array}{l}32 \cdot 3(112) \\
17.9(62) \\
26 \cdot 3(91) \\
2.9(10) \\
20.6(72)\end{array}$ & $\begin{array}{l}23 \cdot 6(59) \star \star \\
20 \cdot 7(52) \\
21 \cdot 6(54) \\
1 \cdot 8(5) \\
32 \cdot 2(81)^{\star \star}\end{array}$ & $\begin{array}{l}26 \cdot 8(292) \\
16 \cdot 0(174) \\
31 \cdot 8(346) \\
4 \cdot 1(45) \\
21 \cdot 3(232)\end{array}$ & $\begin{array}{l}26 \cdot 3(215) \\
19 \cdot 5(160)^{\star} \\
24 \cdot 0(196)^{\star \star} \\
3 \cdot 4(28) \\
26 \cdot 8(219)^{\star \star}\end{array}$ \\
\hline
\end{tabular}

${ }^{\star} \mathrm{p}<0.05 ;{ }^{\star \star} \mathrm{p}<0.01$. Significance values are for sex comparisons at each age level. 
Table 5 Percentage (and frequency) of children who reported an overnight stay in hospital or missed days of school as a function of $M A I S$

\begin{tabular}{|c|c|c|c|c|}
\hline & $M A I S$ & 1: Minor & 2: Moderate & 3: Serious \\
\hline Overnight stay in hospital & $\begin{array}{l}\text { Boys } s^{\star \star \star \star} \\
\text { Girls } \\
\text { Total } \star \star \star \star \star\end{array}$ & $\begin{array}{c}12 \cdot 6(86) \\
9 \cdot 0(44) \\
11 \cdot 1(130)\end{array}$ & $\begin{array}{l}30 \cdot 1(87) \\
19 \cdot 6(42) \\
25 \cdot 7(129)\end{array}$ & $\begin{array}{l}39 \cdot 2(2) \\
42 \cdot 3(1) \\
40 \cdot 2(3)\end{array}$ \\
\hline Missed days of school & $\begin{array}{l}\text { Boys } s^{\star \star \star \star} \\
\text { Girls } \\
\text { Total } \star \star \star \star \star\end{array}$ & $\begin{array}{l}51 \cdot 5(349) \\
49 \cdot 5(244) \\
50 \cdot 7(593)\end{array}$ & $\begin{array}{l}69 \cdot 2(200) \\
66 \cdot 7(142) \\
68 \cdot 1(343)\end{array}$ & $\begin{array}{r}100 \cdot 0(4) \\
42 \cdot 3(1) \\
80 \cdot 5(5)\end{array}$ \\
\hline
\end{tabular}

$\star \star \star \mathrm{p}<0.001 ; \star \star \star \star \mathrm{p}<0.0001$.
This research was funded by a grant from the Scottish Office Home and Health Department.

1 Scottish Office. Scotland's health: a challenge to us all. Edinburgh: HMSO, 1992.

2 Department of Health. The health of the nation: a strategy for health in England. London: HMSO, 1992.

3 Health Education Board for Scotland. Strategic statement. Edinburgh: Health Education Board for Scotland, 1992. 4 Child Accident Prevention Trust. Basic principles of accident prevention: a guide to action. London: CAPT, 1989.

5 Smith $\mathrm{T}$. Accidents, poisoning and violence as a cause of hospital admissions in children. Health (Bull) Edinb, 1990; 49: 237-44.

6 Smith CJ, MacIntosh AM. A review of child accident prevention in Scotland. Glasgow: Centre for Social Marketing, University of Strathclyde, 1994.

7 Read J. The health of the nation and key areas of accidents.

8 Fournal of Health and Safety 1994; 10: 44-8. MD, Bijur PE. The epidemiology of non-fatal injuries among US children and youth. Am f Public Health 1995; 85: 932-8.

to injuries among boys and girls need to be carefully examined.

There is strong internal consistency in responses to the various questions that would not be expected if self report injury data was intrinsically unreliable. Girls reported fewer instances of specific injuries (with the exception of bruises) and also reported experiencing fewer injuries in total than boys. A strong concordance between the various measures of injury severity (MAIS, nights in hospital, and missed days of school) for the medically attended injuries was found.

In sum, we have shown good agreement between self report based national injury estimates and those based on data collected by more conventional means. For adolescent groups self report is preferable, for example, to maternal report. ${ }^{9}$ In addition, a school based survey is an efficient and relatively inexpensive means of collecting a substantial body of injury data in a brief period of time. Whereas there are known sociodemographic biases to hospital admissions and accident and emergency presentation for injury ${ }^{10}$ a large proportion of the school population is captured in a classroom based survey with high response rates.

To conclude, children's self reports of their own injuries are a valid method for obtaining injury incidence data. They are especially useful in understanding injury aetiology because, in addition, children can provide detailed information regarding their social and psychological characteristics as well as the circumstances surrounding injury events. Information of this depth and detail is essential for effective injury prevention but is difficult to obtain from other sources, such as hospital based surveillance systems, which are currently geared more towards identifying service provision needs. 